\title{
La planificación curricular: Punto de partida del trabajo pedagógico
}

\author{
Curriculum planning: \\ Starting point of pedagogical work
}

http://dx.doi.org/10.17981/cultedusoc.13.1.2022.13

Recibido: 7 de enero de 2021. Aceptado: 5 de abril de 2021. Publicado: 15 de enero de 2022 .

\author{
Riky Frank González-Alfaro \\ Universidad Cesar Vallejo. Trujillo (Perú) \\ gonzalezriky213@gmail.com
}

Para citar este artículo:

González-Alfaro, R. (2022). La planificación curricular: Punto de partida del trabajo pedagógico. Cultura, Educación y Sociedad, 13(1), 219-232. DOI: http://dx.doi.org/10.17981/cultedusoc.13.1.2022.13

\section{Resumen}

Introducción: La planificación curricular representa un factor clave para la dinámica educativa, específicamente en los procesos de mediación didáctica-pedagógica. Por tanto, el objetivo del presente artículo es analizar críticamente la producción intelectual y conocer la importancia que tiene la planificación curricular en el logro de aprendizajes significativos en los estudiantes: Metodología: Análisis y discusión bibliográfica que permite sistematizar artículos teóricos y empíricos sobre el tema, donde se considera la revisión de 4 libros de referencia, 16 artículos científicos obtenidos de revistas indexadas en Scielo, Scopus, Redalyc, 6 documentos normativos sobre la educación básica regular y 7 tesis doctorales que abordan la planificación curricular como eje central del trabajo pedagógico. Resultados y Discusión: Se evidencia que la planificación curricular conduce al maestro y sus estudiantes al éxito, al logro de los aprendizajes, y específicamente en los estudiantes al desarrollo de las competencias requeridas para que puedan enfrentar diversas situaciones complejas en su vida diaria. Conclusiones: Es importante que el docente durante la etapa de planificación considere las características del estudiante, así como sus necesidades de aprendizaje, intereses particulares y las particularidades del contexto.

Palabras clave: Planificación de la educación; docencia; aprendizaje; estrategias educativas

\section{Abstract}

Introduction: Curriculum planning represents a key factor for educational dynamics, specifically in the didactic-pedagogical mediation processes. Therefore, the objective of this article is to critically analyze intellectual production and to know the importance of curricular planning in the achievement of significant learning in students: Methodology: Bibliographic analysis and discussion that allows systematizing theoretical and empirical articles on the subject, where the review of 4 reference books is considered, 16 scientific articles obtained from journals indexed in Scielo, Scopus, Redalyc, 6 normative documents on regular basic education and 7 doctoral theses that address curricular planning as the central axis of pedagogical work. Results and Discussion: It is evidenced that curricular planning leads the teacher and his students to success, to the achievement of learning, and specifically in the students to the development of the required competencies so that they can face various complex situations in their daily lives. Conclusions: It is important that the teacher during the planning stage consider the characteristics of the student, as well as their learning needs, particular interests, and the particularities of the context.

Keywords: Educational planning; teaching; learning; educational strategies 


\section{INTRODUCCIÓN}

El aprendizaje de los estudiantes pasa por una serie de procesos y etapas dentro y fuera de la escuela; sin embargo, también deben tener en cuenta: la edad, los ritmos de aprendizaje, el contexto que rodean a cada uno de ellos. El maestro es parte fundamental en la enseñanza y aprendizaje, quien tiene que tomar en cuenta además de lo ya señalado, otros factores y hacer uso de su formación docente, de sus habilidades, destrezas y capacidad de uso de diversas técnicas y estrategias para lograr el fin supremo con sus estudiantes: su aprendizaje. El objetivo es analizar la producción académica referido a la planificación curricular, pero como eje central de la investigación dada la importancia que tiene como punto de partida para el logro de los aprendizajes y el desarrollo de las competencias de los estudiantes. Reiteradamente en las instituciones educativas, los docentes se preocupan más en qué enseñar, por ejemplo, qué contenidos van a abordar en las diferentes áreas académicas, obviando el cómo y con qué enseñar, se deja de lado la planificación de estrategias y actividades que son muy importantes para lograr aprendizajes significativos en los escolares.

El sistema educativo peruano se basa en el Currículo Nacional de Educación Básica-CNEB (2016), cuya característica es el enfoque por competencias, que se viene implementando en las escuelas de manera progresiva en los diferentes niveles educativos. El desarrollo de las competencias en los estudiantes es un desafío para el docente, puesto que tiene que hacer uso de las diferentes prácticas pedagógicas, y para ello es importante y necesario que el maestro tenga que prever todas las acciones para arribar a los resultados deseados, es decir, tiene que elaborar su planificación curricular, donde planteará los momentos de una serie de prácticas pedagógicas como por ejemplo: selección de recursos, material educativo y estrategias, orientaciones para promover evidencias de aprendizaje las que facilitarán visualizar e interpretar las dificultades y progresos del estudiante, formas y técnicas de retroalimentación, prever el uso de los diferentes espacios educativos y organizarlos para favorecer el aprendizaje, plantear preguntas socráticas sobre la validez de sus conclusiones, hipótesis, y para generar el pensamiento crítico, reflexivo, la creatividad; entre otras prácticas pedagógicas (CNEB, 2016).

Si bien es cierto que muchos docentes planifican, prevén las actividades, recursos, estrategias que utilizarán en el aula frente a sus estudiantes, también hay un porcentaje considerable de maestros que trabajan con sus estudiantes de manera empírica, sin tener en cuenta los aspectos ya mencionados de una planificación, y es necesario tratar sobre lo que significa que los maestros realicen una planificación curricular que los conduzca al éxito, al logro de los aprendizajes significativos y desarrollo de las competencias de sus estudiantes. La gestión educativa institucional es muy importante para el óptimo desempeño del docente y que el usuario, en este caso, los estudiantes, queden satisfechos significativamente (Alvarado, 2017).

En los últimos años, el Estado peruano en su afán por mejorar la atención y formación continua del docente, implementó el acompañamiento pedagógico en instituciones educativas focalizadas, donde el docente que requiere apoyo en cuanto a planificación curricular y la ejecución de la misma, estará guiado por un acompañante, con el cual desarrollaran en conjunto las actividades pedagógicas, acompañamiento que permitirá fortalecer sus 
competencias, esto demuestra cuán importante es reorientar los procesos de formación del docente, donde éste sea reflexivo, ético, consciente de su rol como sujeto de cambio (Guerrero, 2018).

El directivo juega un papel muy importante en el desempeño de los docentes, puesto que es el líder pedagógico de la institución y el llamado a monitorear para buscar y recoger información oportuna, confiable, de primera mano, para tomar decisiones oportunas y acompañar a los docentes que lo requieran, para fortalecernos en su planificación y tengan éxitos en sus aulas logrando aprendizajes significativos en los estudiantes (Chuqui, 2019).

En la planificación curricular está el éxito de lograr los aprendizajes esperados, es donde el maestro debe anticipar ,organizar, tomar decisiones oportunas, considerar las aptitudes, contexto y las múltiples posibilidades que propone la pedagogía, estrategias didácticas y los enfoques de las diferentes áreas (Arce, 2018). El docente al planificar tiene que conocer de antemano las competencias, capacidades, desempeños, estándares de aprendizaje según el CNEB para lograr el perfil de egreso de los estudiantes al terminar la educación secundaria; es decir todo lo que concierne al currículo; y para el logro de esto es necesario incluir las estrategias educativas a utilizar, seleccionar los contenidos y las actividades para elaborar experiencias de aprendizaje significativas, teniendo en cuenta los estilos de aprendizaje de los estudiantes (Palés, 2006).

Actualmente para el uso de diferentes estrategias de cómo enseñar para lograr aprendizajes significativos y cómo plasmar dichas estrategias en la planificación del maestro, existen varios enfoques a tomar en cuenta antes de planificar, en los cuales algunos métodos se apoyan en la naturaleza del niño, acuden a las leyes de su constitución pedagógica y desarrollo como individuo; organizar una enseñanza entre pares los ayuda a investigar mejor, esto es propio de las pedagogías activas (López, 2012).

Los resultados de las ultimas evaluaciones censales en el país no son halagadores para la región de Cajamarca, menos para la provincia de Cutervo, donde los niveles de logro "previo al inicio" y "en inicio", abarcan más del 70\% de los estudiantes (Ministerio de EducaciónMINEDU, 2018). Dicha cifra no le hace nada bien a los maestros, y estos resultados se han ido dando a través de los diferentes años en que se ha aplicado esta evaluación censal, razón por la cual la Ley de Reforma Magisterial (Ley 29944, 2013) y su reglamento, resalta la sólida formación que deben tener los docentes para desarrollar eficazmente las diferentes etapas del proceso educativo, como por ejemplo los procesos pedagógicos, las actividades de planificación curricular, la evaluación, entre otros, lo que conducirá a desarrollar las competencias y lograr aprendizajes significativos en los estudiantes.

La planificación curricular siempre ha sido considerada como un factor más dentro de otras áreas, como el desempeño docente, acompañamiento pedagógico y logros de aprendizaje. En ese sentido, y en vías de destacar su importancia para lograr aprendizajes significativos, se realizó el presente estudio, el cual tuvo como objetivo analizar críticamente la producción intelectual sobre la planificación curricular como punto de partida para lograr el desarrollo de competencias y aprendizajes significativos en los estudiantes.

La planificación curricular del maestro debe estar centrada en el enfoque por competencias; para ello es necesario considerar una serie procesos, estrategias y actividades, cuyos resultados deben verse reflejados en los aprendizajes de los estudiantes. Una planificación 
enfocada al desarrollo de las competencias, significa elaborar experiencias de aprendizajes con situaciones significativas retadoras, desafiantes, las cuales pongan en situación al estudiante, que genere el pensamiento crítico, reflexivo, creativo; experiencias que incluyan la autorreflexión del estudiante; que se dé cuenta en pleno proceso de aprendizaje, donde está, cuáles son los obstáculos que tiene para lograr su objetivo y como puede superarlos. Se requiere una planificación que oriente al estudiante a desarrollar su creatividad para resolver problemas de su entorno, que proponga diversas alternativas de solución, y donde el docente haga uso de la evaluación formativa, el acompañamiento, la retroalimentación. Solo este tipo de planificación logrará desarrollar las competencias en los estudiantes, tan necesarias en el mundo actual, para enfrentar los diversos problemas que atañen a la sociedad.

\section{MetodoloGía}

Este trabajo de investigación bibliográfica es una síntesis teórica y argumentativa de múltiples aportes pedagógicos, brindando una interpretación descriptiva del proceso de planificación curricular y su importancia en el trabajo docente. La metodología usada es de un estudio de tipo documental. Se cimienta sobre la revisión organizada, exploratoria, amplia y crítica de distintas fuentes documentales con un proceso de análisis científico, aplicando criterios de selección sobre lo más importante de la temática a tratar, partiendo de una revisión sistemática usando el protocolo prisma en su diagrama de flujo.

La operacionalización de la investigación se realizó partiendo de la identificación, el registro y recopilación de 230 artículos científicos indexadas en Scielo, Scopus, Redalyc, utilizando palabras clave que son: a) planificación curricular b) currículo c) acompañamiento pedagógico y planificación curricular d) importancia de la planificación curricular e) Desarrollo de competencias f) Educación y planificación g) currículo nacional f) proyecto educativo nacional y también de libros virtuales. Luego se hizo una revisión analítica del total de fuentes seleccionándose 100, las cuales fueron analizadas de manera específica según la variable de estudio para establecer coincidencias, similitudes o diferencias entre uno y otro; seleccionándose finalmente 38 fuentes documentales, que fueron evaluados para la elegibilidad, descartándose de todos ellos cuatro, porque no mostraban y no respondían a resultados explícitos de la temática tratada.

Finalmente, se incluyó en la revisión bibliográfica treinta y tres fuentes documentales de acuerdo a criterios de relevancia antes mencionado para alcanzar el propósito del presente estudio con ciertos procesos que aseguren la confiablidad y validez en la manifestación de resultados y que conforman el corpus de análisis del presente, y que fueron organizadas y archivadas en el gestor de referencias bibliográficas y documentos Mendeley, la que nos garantiza la confiabilidad de la información recopilada en este trabajo de investigación.

\section{Resultados y Discusión}

En función de la revisón teórica y análisis exhaustivo de la literatura científica se presenta la construcción argumentativa relacionada con las categorías centrales del estudio: 


\section{Preparación para el aprendizaje de los estudiantes}

Cuando el maestro habla de aprendizaje en el estudiante, se refiere a su cambio conductual permanente, tal como manifiesta Schunk (2012), que el aprendizaje es el cambio en la conducta de una persona, es decir, cuando se vuelve capaz de hacer algo distinto a lo que hacía antes. Según el Marco de Buen Desempeño Docente (MBDD, 2012), el maestro tiene que prepararse antes de enseñar a sus estudiantes, tiene que prever una serie de actividades antes de llegar al aula, como el conocer a sus estudiantes, su contexto sociocultural, elaborar colegiadamente su planificación curricular como la programación anual, sus unidades didácticas o proyectos, sus sesiones o experiencias de aprendizaje; éstas deben buscar el desarrollo de las competencias, poner en sinergia las diversas capacidades al mismo tiempo para el logro de aprendizajes significativos, además de plasmar una serie de estrategias, técnicas, recursos educativos que utilizará a lo largo de su labor. Así mismo dentro de esta preparación para el aprendizaje, el maestro debe tener dominio disciplinar, estar constantemente actualizándose, leyendo, investigando sobre lo que va a enseñar, conocer y aplicar la evaluación formativa con sus estudiantes.

En su artículo de investigación sobre la evaluación del desempeño docente, Gálvez y Milla (2018) concluyen que también es de vital importancia que los maestros sepan manejar los procesos cognitivos: recepcionar y procesar la información adecuadamente, y el manejo de los procesos pedagógicos, todo ello facilitará el aprendizaje del estudiante. Bixio (2003) propone una planificación basada en una estrategia de didáctica secuencial donde cada una de ellas será secuenciada en el año lectivo donde se tendrá en cuenta poner los datos esenciales como por ejemplo, el docente mencionará cuáles serán las estrategias didácticas que utilizará, y estrategias de aprendizaje del estudiante.

El maestro es uno de los actores principales para que los estudiantes logren el perfil de egreso al culminar la Enfoque Basado en Riesgo-EBR, tal como lo señala la Ley General de Educación (2003, art. 56), señala que el maestro es parte fundamental en el proceso de aprendizaje, debe mostrar idoneidad en su profesión correspondiéndole planificar las diferentes actividades para el logro de aprendizajes significativos en los alumnos. Así mismo, el desarrollo de aprendizajes fundamentales en los estudiantes es de imperiosa necesidad, tal como lo señala el Proyecto Educativo Nacional-PEN (2020) al 2021, éstos los preparan para el saber ser, saber hacer, saber conocer y saber convivir y para desarrollarlos de manera eficaz, creativa y con responsabilidad, los maestros deben estar preparados para lograrlo.

El Estado en su afán por formar estudiantes competentes, capaces de actuar con sentido ético y con eficacia ante situaciones problemáticas desde el 2017 está implementando el enfoque por competencias en el país, tal es así que el CNEB (2016) da las orientaciones pertinentes para que los docentes tomen en cuenta en su planificación curricular, ejecución y evaluación de los procesos de E-A en los diferentes espacios educativos. Dentro de estas orientaciones a tomar en cuenta en su planificación curricular el de proponer situaciones significativas retadoras, que generaran interés en los estudiantes, desarrollar pensamiento crítico, creatividad y situaciones que sean de interés (CNEB, 2016). Esto está concatenado con las características que debe tener el maestro, tal como está señalado en el MBDD (2012) donde se define los dominios, las competencias y los desempeños que deben 
caracterizar a un buen docente; menciona que la labor como maestro es importante en el cumplimiento de la misión de la escuela, para ello es valioso, que si queremos lograr la escuela que queremos, ello dependerá que nosotros entendamos lo importante que es la planificación y desarrollo de la enseñanza, para lograr grandes aprendizajes en nuestros estudiantes.

\section{Preparación para la enseñanza del aprendizaje}

Los maestros, además de prepararse para el aprendizaje, para lo cual deben planificar, tienen que hacer efectiva esa planificación, y esto se da, según el MBDD (2012) durante el manejo y desarrollo de la experiencia de aprendizaje, teniendo en cuenta los enfoques transversales, acompañamiento en la gestión del aprendizaje dentro de un clima favorable, manejo disciplinar, la motivación permanente, desarrollo y uso de estrategias metodológicas y el uso de instrumentos para una evaluación formativa que identificarán el logro de los retos en el proceso de aprendizaje. Así mismo la planificación tiene como objetivo primordial garantizar la cobertura curricular y preparar la enseñanza en correspondencia con las descripciones curriculares y las necesidades que surgirán durante el proceso de enseñanza-aprendizaje (Amaro, 2011).

El saber de un maestro constituye un saber social por que trabaja con individuos que conforman la sociedad, no trabaja con objetos, sino con sujetos y en función a un objetivo: transformar a los estudiantes, educarlos y enseñar, el actuar con otras personas, que saben que les enseño, que saben que es un maestro, por lo tanto, el saber se manifiesta a través de una relación compleja entre maestro y estudiantes (Tardiff, 2013). Este saber social concuerda con lo que afirma Niemeyer (2006) que la dimensión social del aprendizaje está por encima de la dimensión individual.

Para Bixio (2003), después de haber planificado las secuencias didácticas, estas se desarrollarán a través de diferentes instancias las cuales incluyen actividades de enseñanzaaprendizaje, cuyos aspectos son:

- Qué: Comprende lo conceptual.

- Cómo: Que alude a lo procedimental.

- Con Qué: Alusivo a los recursos que utilizaremos y la bibliografía a usar.

- Con Quién: Si será un trabajo en grupo o individual.

- Cuando: Que tiene que ver al tiempo que se ha previsto para la estrategia didáctica.

- Dónde: Espacio donde se ejecutará la actividad, puede ser en el aula o fuera de ella.

\section{Dimensión pedagógica del docente}

Esta dimensión constituye el centro del saber profesional del docente, el cual se ha ido construyendo a base de la reflexión entre la teoría y práctica donde haciendo uso de sus diversos saberes logra cumplir a cabalidad su función como docente. Esta dimensión está referida específicamente a la práctica de la enseñanza, en la cual el docente tiene que manejar diversas estrategias y saberes para lograr que el estudiante se interese por el 
aprendizaje y asuma la responsabilidad para aprender y formarse. Así mismo requiere la presencia constante de la ética en el docente para educar (MBDD, 2012). El docente desde que empieza su formación necesita conocer y ejercer una competencia docente completa en relación a la claridad de la planificación de una asignatura, conteniendo el uso del tiempo y la innovación curricular, porque planificar también implica tomar en cuenta la realidad presente, sin olvidar que ésta planeación tiene una intención retrospectiva (el pasado simboliza evaluar) y prospectiva (establecer los fines educativos), es aquí donde el maestro tiene la oportunidad de innovar, transformar, mejorar su propia práctica, haciendo uso de sus saber experiencial (López y Villanueva, 2019).

Así mismo, para Krichesky (citada por la Fundación Navarro Viola, 2017), la dimensión pedagógica no sólo debe estar presente en el docente, sino también en los directivos del colegio, para que de verdad allí se aprenda; y esta dimensión en el director se construye a partir de asumir el rol de asesor de sus docentes, a quienes monitoreará y acompañará en su práctica pedagógico, desarrollando un trabajo colaborativo entre director y docente. El directivo es pieza clave para que los docentes desarrollen y potencialicen esta dimensión de su actuación pedagógica donde los maestros deben saber y deben saber hacer, así como las actitudes propias de su condición docente (Castañeda, 2019).

Según el MBBD (2012), aquí pueden distinguirse como mínimo los siguientes aspectos:

1. Juicio pedagógico: Referido a como los docentes deben tener diversos criterios para entender las múltiples necesidades de aprendizaje que tienen los estudiantes. Así mismo tener en cuenta sus estilos de aprendizaje, asumir el acompañamiento en el momento indicado y valorar los avances que tiene a lo largo del proceso de aprendizaje.

2. Liderazgo Motivacional: El maestro debe tener la capacidad de generar interés, motivación por aprender del estudiante, esto dentro de una heterogeneidad de edades, perspectivas y particularidades; y darles confianza para que logren el desarrollo de todas sus competencias aún en circunstancias adversas, hacer uso de la resiliencia.

3. Vinculación: El maestro no puede estar exento de mantener lazos de afectividad docente-estudiante. Es importante el manejo de las habilidades blandas, uso de la inteligencia emocional, hacerle sentir al estudiante que están siendo acompañados no sólo en su aprendizaje, sino también en el cuidado de su estado emocional.

\section{Planificación curricular}

Según Chávez (2018) la planificación curricular constituye una parte fundamental en el proceso de prever qué y cómo se llevará los aprendizajes de los estudiantes en las aulas. Tal como dice Kaufman (2001) que planificar el currículo es encargarse de establecer que debe hacerse, prever que se va a hacer, para que luego se tomen decisiones prácticas para su implantación.

Ander-Egg (1991) define a la planificación curricular como la labor de hacer uso de una serie de procedimientos a través de los cuales se organizan una serie de actividades las cuales serán usadas para lograr los objetivos planteados, hacer que ocurran cosas que de otro modo, no ocurrirían. Montilla (2015) también refuerza la concepción de Ander-Egg (1991), y sostiene que la planificación es una metodología que usa el docente en su tra- 
bajo, para que pueda tomar decisiones oportunas y efectivas en el proceso de enseñanza y aprendizaje.

El logro de los aprendizajes en los estudiantes es una tarea de gran responsabilidad que recae en el maestro, para ello es necesario que prevea la manera de poder lograrlo mediante la elaboración de una planificación curricular con coherencia entre el plan de estudios contenidos programados y el perfil de egreso de los estudiantes de la EBR. Ande-Egg (citado por Chávez, 2018) define a la planificación curricular como la labor de hacer uso de varios de procedimientos a través de los cuales se organizan una serie de actividades previstas con anterioridad y que serán usadas para lograr los objetivos planteados. Montilla (2015) concibe la planificación curricular como una metodología que usa el docente en su trabajo, para que pueda tomar decisiones oportunas y efectivas en el proceso de enseñanza y aprendizaje.

Para lograr los aprendizajes significativos, es necesario que el docente tenga en cuenta en su planificación no solo los propósitos de aprendizaje, sino también, el enfoque, las teorías pedagógicas que sustentaran su trabajo, y para ello, debe estar preparado, apto, para una planificación curricular idónea, donde el estudiante desarrolle su inteligencia y construya sus conocimientos en acción y en situación y por la reflexión sobre la acción y sus resultados (Masciotra, 2018). La persona aprehende y comprende las situaciones nuevas a través de lo que ya sabe y modifica sus conocimientos anteriores a fin de acomodarse. Cada acomodación a una situación permite ampliar y enriquecer la red de conocimientos anteriores de los cuales dispone una persona, y esta progresión continua de la red le permite tratar situaciones cada vez más complejas.

El Ministerio de Educación del Perú, a través del programa curricular de secundaria, concibe a la planificación, como el arte de imaginar y delinear procesos para que los estudiantes logren aprender, es decir lograr desarrollar las competencias y capacidades propuestas en el perfil de egreso del CNEB, así mismo considera vital, que se debe tener en cuenta las necesidades de aprendizaje, aptitudes, contexto, experiencias, intereses de los estudiantes y que al momento de planificar se debe tener muy claro el propósito de aprendizaje, donde están inmersos, las competencias, capacidades, desempeños y estándares de aprendizaje, según el grado, área y nivel educativo. Desarrollar la competencia significa que el estudiante tiene "un saber actuar complejo que se apoya sobre la movilización y la utilización eficaces de una variedad de recursos" (Tardiff, 2013).

Córdova (2019) realizó un trabajo de investigación sobre desempeño docente y resultado de los aprendizajes de los estudiantes de las instituciones educativas de Huancayo, donde concluyeron que existía una relación directa entre las variables de desempeño docente y aprendizajes de los estudiante, donde el desempeño docente estaba referido a la preparación del maestro para la enseñanza, donde está inmerso la planificación curricular, producto de la buena formación y constante actualización coherente con las exigencias educativas de hoy.

Bautista (2019) realizó un trabajo de investigación sobre el desarrollo del desempeño docente de matemáticas a través del monitoreo, acompañamiento e interaprendizaje en el colegio "San Carlos" para el 2018 en la ciudad de Puno y determinó que el monitoreo y acompañamiento a los docentes, influye positivamente en cuanto al dominio sobre la preparación para el aprendizaje, es decir sobre la planificación curricular. Así mismo, Salazar 
(2018) en su investigación sobre la influencia de la capacitación docente en la planificación y evaluación curricular en los docentes de la Universidad Las Américas en Perú, concluye que, al desarrollarse dicho evento, mejoró significativamente la planificación curricular de los docentes de esa casa de estudios.

Meléndez y Gómez (2008) realizaron una investigación en las escuelas robinsonianas del municipio de Iribarren en el estado de Lara en Venezuela, y concluyó que la planificación de los docentes de estas instituciones tenía serias deficiencias, y para poder subsanarlas, elaboró un modelo de guía de planificación curricular en el aula, basado en el enfoque por competencias. Así mismo, recalca la función del maestro como mediador de los aprendizajes, el encargado de planificar, pero de acuerdo con las necesidades de los estudiantes para que estén preparados para hacer frente a los diferentes retos que se presente en su vida. A la par de lo que dice Meléndez y Gómez (2008), Bixio (2003) también resalta que, para el logro de aprendizajes, significativos, los docentes deben estar preparados, actualizados, desarrollando una planificación teniendo en cuenta los momentos vinculantes más importantes como son: análisis o diagnóstico, propósito, elección de las diversas estrategias de metodología, las herramientas y evaluación de la planificación. Quiere decir entonces que, cumpliendo estos momentos, se logrará una verdadera planificación, basado en el enfoque por competencias desarrollando las fases siguientes: contextualización, teorización, desarrollo, problematización y demostración de la competencia (Bixio, 2003).

Debemos reconocer que la docencia es un trabajo complejo, requiere de muchas acciones por parte del docente, acciones de reflexión, de ética, de compromiso, de preparación, planificación para los aprendizajes de sus estudiantes, dentro de un contexto determinado, será el guía y mediador de estos aprendizajes, lo que se le hará ver como un agente de cambio, pues sabe la importancia la interacción que tiene con sus alumnos, del poder de sus palabras, pero al mismo tiempo, sabe la importancia de la actuación y planificación colegiada con sus pares, esto está refrendado por el MBDD (2012) que menciona que la sociedad espera que los maestros preparen a las futuras generaciones para enfrentar los grandes retos que día a día se presentan y concretizar los aprendizajes fundamentales del estudiantado, se requiere de una gran transformación, compromiso y cambios de las prácticas pedagógicas, una planificación para los aprendizajes, romper con esquemas de enseñanza tradicional, o improvisada, para llegar a la producción de conocimiento.

Consolidando lo referido por los diversos autores, la planificación curricular dentro del trabajo pedagógico, es el punto de partida para el logro de los aprendizajes, es vital, sin ello, es imposible conseguir el desarrollo de las competencias, una interacción entre los estudiantes que genere aprendizajes significativos, habrá ausencia de situaciones significativas, retadores que promuevan y conduzcan a los estudiantes a desarrollar el pensamiento crítico, complejo, la creatividad; la planificación curricular orienta al maestro a encaminar su práctica pedagógica en el aula o fuera de este, le ayuda a tomar decisiones oportunas y efectivas en su labor. De la misma forma, los maestros deben concientizarse que enseña, como enseña y tomar la realidad, el contexto donde se desarrollará el aprendizaje del estudiante y que estas acciones y operaciones deben estar sistematizadas (Pérez, Valdés y Garriga, 2019).

Las diferentes posturas, muestran que la efectividad de la escuela, en cuanto a los aprendizajes efectivos está determinado por la calidad del maestro en su trabajo pedagógico en 
el aula, el dominio disciplinar que tiene, la habilidad, metodología, entendimiento de saber enseñar a estudiantes de diferentes contextos socio-familiares y los recursos educativos que hace uso.

Para el logro de las competencias y capacidades es necesario, según el CNEB (2016) se debe organizar de manera secuencial las diferentes actividades de aprendizaje, pronosticadas en la programación anual; es aquí donde se especificará que se quiere lograr, como se va logrando, como se evaluará, los tiempos para cada actividad y los materiales que se utilizarán. Por lo tanto, se necesita que el maestro prevea todas las acciones necesarias para lograr aprendizajes significativos, es el momento en que el maestro rompa esos esquemas tradicionales de planificar y lo haga de manera vivencial y flexible para que pueda lograr aprendizajes en sus estudiantes que enfrenten los diferentes retos que presenta la sociedad.

Así mismo se debe tener en cuenta, que los 3 primeros objetivos del PEN (2020) están orientados al logro de aprendizajes pertinentes que desarrollan las competencias para enfrentar los diferentes desafíos de su realidad, así como aprendizajes de igual calidad para todos, pero esto se logrará con docentes con desempeño comprometidos con la educación, que hagan uso de sus saberes para preparar el aprendizaje de sus estudiantes, con una planificación coherente y acorde con las necesidades de aprendizaje de sus estudiantes.

Los docentes para una planificación efectiva es necesario que conozcan los diferentes modelos, ante lo cual, Bolaños y Molina (2007) consideran que existen 3 modelos de planificación curricular:

1. Modelos lineales, caracterizados por una relación directa de reacción en sucesión, tal es el ejemplo que cuando planteamos objetivos estos van a derivar a varios contenidos que para desarrollarlos se utilizarán determinadas estrategias.

2. Modelos sistemáticos, referido a la relación entre macros sistemas, como por ejemplo el sistema social y los subsistemas que lo componen, como el currículo y el proceso curricular le dará sentido en tanto cumpla con las expectativas de las intencionalidades sociales.

3. Modelos integradores, donde los elementos del currículo muestran relaciones recíprocas, dentro de un proceso interactivo y permanente, ejemplo de este tipo de modelo es que cuando planteamos objetivos, estos van a determinar contenidos específicos y estrategias metodológicas, y éstas a su vez condicionarán los ambientes, recursos, materiales, etc., que serán de utilidad para lograr los objetivos.

Los maestros deben estar siempre a la vanguardia de la educación, estar constantemente investigando, actualizándose, participar de capacitaciones reales, verdaderas, no donde se contrate a cuatro educadores conocedores de temas educativos, pero que disertan para cientos de maestros al mismo tiempo, y luego los certifican; eso no es capacitación, es una falsedad, pues una verdadera capacitación implica que el maestro en ese proceso de capacitación, analice, reflexione, descubra los obstáculos que tiene en su trabajo, los retos que se le presentan y la firme decisión de enfrentarlos (Freire, 1993). Un docente que planifique en función al propósito de aprendizaje, donde el estudiante internalice que ser competente implica ponerse en situación, analizar la situación problemática y reflexionar sobre las opciones que tiene para resolverla. 
Para que los docentes puedan lograr una planificación acorde con el enfoque por competencias, como lo pide el Ministerio de Educación, es necesario que éstos se empoderen en este proceso de planificar que es tan importante, para esto, es necesario que se involucren en diferentes actividades que contribuyan a su formación, como por ejemplo, la auto capacitación, la participación en Grupos de Interaprendizaje-GIA, asistencia a talleres sobre planificación curricular, por lo que los maestros al planificar tendrán en cuenta el enfoque por competencias, y allí descubrirá que para abordarlas, existen muchas formas, Así mismo las competencias son procesos complejos de desempeño con idoneidad en determinados contextos, integrando diferentes saberes, saber: ser, hacer, conocer y convivir. Educar en competencias requiere asumir una nueva comprensión y raciocinio que vaya más allá de la parcelación, para que aborde la realidad en toda su dimensionalidad (Tobón, 2004).

El maestro de hoy tiene que ser muy diferente al del pasado, tiene que ser un maestro preguntón, que invite al estudiante a analizar, reflexionar, organizar, crear, organizar conocimientos y donde el docente ya no es un facilitador que lo sabe todo ni lo tiene que resolver todo sino un motivador, un mediador de experiencias únicas, que provoque la discusión crítica, que su planificación esté lleno de situaciones significativas retadoras, que aprende de su mismo contexto que lo rodea, desde su propia realidad de lo que está vivenciando, logrando aprendizajes significativos, para ello es necesario tener siempre presente que la planificación es antes y durante la ejecución del mismo y que es el mismo docente que debe realizarlo, no debemos propiciar el fracaso de la planificación que no viene a ser sino cuando unos planifican y otros le ejecutan, porque se corre el riesgo, que casi es siempre, que los últimos no se sienten comprometidos con lo que ellos no planificaron y terminan desarrollando otra cosa, menos lo planificado por otros (Navarro, Pereira, Pereira y Fonseca, 2010).

El esfuerzo que hace cada estudiante para lograr sus aprendizajes no excluye la mediación docente o el apoyo de sus compañeros conseguirlo, la distancia entre lo que el estudiante aprende por su propio medio y lo que aprende con el apoyo docente, es lo que se denomina zona de desarrollo próximo, por lo que esto implica que el maestro debe tener criterio para saber qué enseñar, cómo enseñar y cuándo enseñar (Ander-Egg, 1993). Es preocupante también que los maestros al planificar dejan de lado el Currículo de Educación Básica Regular enfocado al desarrollo de las competencias y se centran en pensar en lo que deberían enseñar. Al planificar se debe tomar en cuenta el perfil de egreso de los estudiantes al culminar la EBR, los estándares de aprendizaje, las competencias y capacidades, para que en base a ello planifique todas las actividades orientadas a lograr aprendizajes significativos (Regalado, 2020).

\section{CONCLUSIONES}

Se exponen las siguientes ideas conclusivas:

La planificación curricular es el punto de partida para el logro de aprendizajes significativos y el desarrollo de las competencias y capacidades en los estudiantes.

Es importante que el docente durante la etapa de planificación tenga en cuenta las características de sus estudiantes, su contexto, sus necesidades de aprendizaje, los intereses que tienen, así como los recursos a utilizar. 
Es importante la planificación curricular dentro del trabajo pedagógico del maestro para pasar de la teoría a la práctica.

Mientras mejor esté preparado el docente, mayor será las posibilidades de éxito que tendrá con sus estudiantes en cuanto al logro de aprendizajes significativos.

La planificación curricular implica innovar, transformar y mejorar la práctica pedagógica.

\section{REFERENCIAS}

Alvarado, M. P. (2017). Gestión Educativa Institucional y Gestión de recursos humanos en la satisfacción del usuario en una institución educativa, Lima-2017. [Tesis para grado]. Univesidad César Vallejo, Lima, Perú. Disponible en https://hdl.handle. net/20.500.12692/13004

Amaro, R. (2011). La Planificación Didáctica y el Diseño Instruccional en Ambientes Virtuales. Investigación y Post grado, 26(2), 129-160. Disponible en https://revistas.upel. edu.ve/index.php/revinpost/article/view/1455

Ander-Egg, E. (1993). La planificación educativa. Buenos Aires: Magisterio del Río de la Plata.

Ander-Egg, E. (1991). Introduccion a la planificación. México, D.F.: Siglo XXI.

Arce, H. R. (2018). Percepciones de los docentes del nivel primaria de una institucion educativa de la UGEL-04 sobre Estrategia de Acompañamineto Pedagógico de la Educación Basica Regular. [Tesis de grado]. Pontificia Universidad Católica del Perú, Lima, Perú. Disponible en http://hdl.handle.net/20.500.12404/15605

Bautista, J. (2019). Desarrollo del Desempeño Docente de Matemática a través del monitoreo acompañamiento e interaprendizaje. [Tesis grado]. Universidad Nacional del Altiplano, Puno, Perú. Disponible en http://repositorio.unap.edu.pe/handle/UNAP/12150

Bixio, C. (2003). Como planificar y evaluar en el aula. propuestas y ejemplos. Seattle: Amazon Digital Services.

Bolaños, G. y Molina, Z. (2007). Introducción al currículo. Costa Rica: Euned.

Castañeda, M. (2019). Del conocimiento histórico al conocimiento escolar.Planificación docente para la enseñanza de Historia y Ciencias Sociales. Historia y Memoria, (18), 289-313. https://doi.org/10.19053/20275137.n18.2019.7447

Chávez, S. (2018). La planificación curricular y su relación con el desempeño docente en la institución educativa No 82939, Bambamarca. [Tesis de grado]. Universidad Cesar Vallejo, Lima, Perú. Disponible en https://hdl.handle.net/20.500.12692/34522

Chuqui, M. L. (2019). Influencia del monitoreo y acompañamiento pedagógico implementado por el director, en la mejora del desempeño docente en las Instituciones Educativas Mercedarias Misioneras de Lima. [Tesis de maestría]. Universidad Antonio Ruiz de Montoya, Lima, Perú. Disponible en http://repositorio.uarm.edu.pe/handle/ UNIARM/1997 
Córdova, M. (2019). Desempeño docente y resultados de los aprendizajes en estudiantes de instituciones educativas secundarias de Huancayo. [Tesis doctorado]. UNCP, Huancayo, Perú. Disponible en http://hdl.handle.net/20.500.12894/5420

Freire, P. (1993). Educacion popular. Buenos Aires: Clacso.

Fundación Navarro Viola. (diciembre 1, 2017). Graciela Krichesky - La dimensión pedagógica Graciela Krichesky - La dimensión pedagógica de la tarea del director: la observación de clases. Dimensión Pedagógica. Disponible en https://educar.pe/gracielakrichesky-la-dimension-pedagogica/

Gálvez, E. y Milla, R. (2018). Evaluación del desempeño docente: Preparación para el aprendizaje de los estudiantes en el Marco de Buen Desempeño Docente. Propósitos y Representaciones, 6(2), 407-429. http://dx.doi.org/10.20511/pyr2018.v6n2.236

Guerrero, G. M. (2018). Acompañamiento pedagógico y reflexión crítica docente en la Unidad Educativa Fiscal Teniente Hugo Ortiz - Ecuador 2018. Logos, 8(1), 1-29. Disponible en https://revistas.uap.edu.pe/ojs/index.php/LOGOS/article/view/1589

Kaufman, R. (2001). Planificación de los sitemas educativos. México, D.F.: Trillas.

López, L. I. y Villanueva, O. E. (2019). La atención selectiva del docente en los procesos de planeación curricular, aprendizaje y evaluación. Diálogos sobre educación, 19(10), 1-23. https://doi.org/10.32870/dse.v0i19.489

López, M. A. (2012). Aportes de la pedagogía activa a la educación. Plumilla Educativa, 4(1), 33-42. https://doi.org/10.30554/plumillaedu.4.605.2007

Masciotra, D. (2018). La compétence : entre le savoir agir et l'agir réel. Perspective de l'énaction. Éthique publique, 19(1). https://doi.org/10.4000/ethiquepublique.2888

Meléndez, S. y Gómez, L. (2008). La planificación curricular en el aula. Un modelo de enseñanza por competencias. Laurus, 14(26), 367-392. Disponible en https://revistas. upel.edu.ve/index.php/laurus/index

Montilla, Y. (Mayo 1, 2015). Articulo Planificación Curricular. [Trabajo Maestría]. Disponible en https://maestriagerenciamerida.wordpress.com/category/articulo-planificacion-curricular-yasmin-montilla/

Navarro, Y., Pereira, M., Pereira, L. y Fonseca, N. (2010). Una mirada a la planificación estratégica curricular. Telos: Revista de Estudios Interdisciplinarios en Ciencias Sociales, 12(2), 202-216. Disponible en http://ojs.urbe.edu/index.php/telos/article/view/1829

Niemeyer, B. (2006). El aprendizaje situado: una oportunidad para escapar del enfoque del déficit. Revista de educación, 341, 99-122. Disponible en https://www.educacionyfp.gob.es/revista-de-educacion/numeros-revista-educacion/numeros-anteriores/2006/ re341/re341-05.html

Palés, J. L. (2006). Planificar un Currículum. Educacion Medica, 9(2), 59-65. http:// dx.doi.org/10.4321/S1575-18132006000200004

Pérez, A., Valdés, M. B. y Garriga, A. T. (2019). Estrategia didáctica para enseñar a planificar los procesos de enseñanza y aprendizaje de la matemática. Revista Educación, 43(2), 1-18. https://doi.org/10.15517/revedu.v43i2.32236 
Regalado, Y. (2020). Talleres de implementación pedagógica del Currículo Nacionalen os procesos didacticos de la competencia lectora. Educacion Básica Regular-Ancahs 2019. [Tesis doctoral]. Universidad César Vallejo, Chimbote, Perú. Disponible en https://hdl. handle.net/20.500.12692/43389

República de Perú. Consejo Nacional de Educacion y MINEDU. (2020). Proyecto Educativo Nacional —PEN 2036 - Lima: Consejo Nacional de Educación. Disponible en https://hdl.handle.net/20.500.12799/7661

República de Perú. MINEDU. (2018). ECE ¿Qué aprendizajes logran nuestros estudiantes? Lima: MINEDU. Recuperado de http://umc.minedu.gob.pe/wp-content/ uploads/2018/10/Informe-Nacional-ECE-2018.pdf

República de Perú. MINEDU. (2016). Currículo Nacional de Educación Básica-CNEB. [Resolución Ministerial No. 281]. Disponible en http://www.minedu.gob.pe/curriculo/

República de Perú. MINEDU. (2013). Ley de reforma magisterial. [Ley No. 29944]. Lima: MINEDU. Disponible en https://www.minedu.gob.pe/reforma-magisterial/ley-reforma-magisterial.php

República de Perú. MINEDU. (2012). Marco del Buen Desempeño Docente-MBDD. [Online]. Recuperado de http://www.minedu.gob.pe/pdf/ed/marco-de-buen-desempeno-docente.pdf

República de Perú. MINEDU. (29 de julio de 2003). Ley General de Educación. [Ley No. 28044]. Disponible en https://www.gob.pe/institucion/congreso-de-la-republica/normas-legales/118378-28044

Salazar, S. (2018). Influencia de la capacitación docente en la planificación y evaluación curricular en los docentes de la Universidad Las Américas, 2016. [Tesis grado]. Universidad Nacional de Educación, Lima, Perú. Disponible en http://repositorio.une.edu. pe/handle/UNE/2491

Schunk, D. H. (2012). Teorias del aprendizaje. [6 ed.]. México, D:F.: Pearson.

Tardiff, A. (2013). Los saberes del docente y su Desarrollo Profesional. Madrid: Narcea.

Tobón, S. (2004). Formación Basada en Competencias. Madrid: Ecoe ediciones. Available from https://www.uv.mx/psicologia/files/2015/07/Tobon-S.-Formacion-basada-en-competencias.pdf

Riky Frank Alfaro González es Profesional en educación secundaria; Especialista en ciencias sociales; Magister en gestión educativa. 27 años de experiencia en docencia en colegios del área rural y urbana. ORCID: https://orcid.org/0000-0002-1699-0374 